\title{
OPPORTUNISTIC INTESTINAL PROTOZOAN PARASITIC INFECTION IN HIV POSITIVE PATIENT IN JAMNAGAR, GUJARAT
}

\author{
Gupta M. ${ }^{1}$, Sinha M. ${ }^{2}$, Raizada N. ${ }^{3}$ \\ ${ }^{1}$ Department of Microbiology, UCMS \& GTB Hospital, Delhi. \\ ${ }^{2}$ Department of Microbiology, M.P. Shah Medical College, Jamnagar, Gujarat. \\ ${ }^{3}$ Department of Community Medicine, M.P. Shah Medical College, Jamnagar, Gujarat.
}

\begin{abstract}
The case definition of AIDS encompasses a spectrum of infections and malignancies, labelled as opportunistic infections. In recent years, numerous studies have outlined the emergence of opportunistic gastrointestinal protozoa that have caused diarrhoeal illness among HIV - AIDS patients. Purpose of present study was to determine the prevalence of opportunistic intestinal protozoal parasites in HIV positive patients with or without diarrhoea. A total of 100 stool samples of HIV positive patients were examined for protozoal parasites by microscopy. Protozoal parasites were detected in 25 HIV positive patients; in $41.37 \%$ of patients with diarrhoea and in $2.38 \%$ of patients without diarrhoea. Isospora belli appeared to be a predominant parasite associated with diarrhoea among HIV patients. Cryptosporidium revealed of its asymptomatic carriage along with its association with acute and chronic diarrhoea. Prevalence of Microsporidia and Cyclospora cayetenensis was found to be very low.
\end{abstract}

Key words: HIV, diarrhoea, opportunistic protozoa.

\section{Introduction}

The progressive destruction of immune system by chronic HIV infection leading to progressive fall in level of CD 4 cells $(<200$ to<50) is known to be responsible for the occurrence of infections by all types of opportunistic micro - organisms in HIV infected individuals. Protozoal parasites that cause mild to self limited disease in immunocompetant host can cause prolonged, intractable, recurrent and severe diarrhoea in HIV patients inducing weight loss and cachexia. Numerous studies have

\section{Correspondence to:}

Dr. Monika Gupta

Department of Microbiology

UCMS \& GTB Hospital, Delhi.

E-mail: raizada_monika@yahoo.co.in outlined the emergence of important gastrointestinal protozoa like Microsporidia $\mathrm{sp}$, Cryptosporidium sp, Isospora belli and Cyclospora cayetenensis., ${ }^{1,2}$ Besides these, HIV infected individuals also develop infection with Giardia lamblia ${ }^{3}$ and Entamoeba histolytica. ${ }^{4}$ Studies have highlighted Cryptosporidium as the predominant pathogen with significant association to diarrhoeal cases 5 along with high prevalence of asymptomatic carrier status among HIV positive individuals. Latent infections can be reactivated and may lead to active diseases later when the immunity of patient diminishes further.

Isospora belli usually results in protracted and some times profuse diarrhoeal disease (in the absence of appropriate antibiotic therapy) in AIDS patient. ${ }^{6}$ Studies on intestinal microsporidiosis from developed countries have revealed $6-60 \%$ prevalence 
among AIDS patient. ${ }^{2}$ Cyclosporiasis has been reported with increasing frequency in United States, Latin America, Nepal, Peru \& New Guinea. The disease usually presents with watery diarrhoea of $19-43$ days $7,8,9$, if diarrhoea in AIDS patients. ${ }^{7}$ Since the diarrhoea due to parasitic aetiology among HIV patient is on rise during recent times as the reports are being available increasingly with time, the present study was undertaken to examine the prevalence of opportunistic enteric parasites among HIV patients attending G.G.S. Hospital, Jamnagar, Gujarat.

\section{Methods}

A cross-sectional study was carried out in 100 known HIV infected cases attending Guru Gobind Singh Hospital, Jamnagar during 2002 (January - December) in the Department of Microbiology. All known HIV positive patients consenting to give two consecutive stool samples were included in the study. The HIV infected clients included in the study were divided into two groups on the basis of absence or presence of diarrhoea. The clients with diarrhoea were further sub-divided on the basis presence of acute or chronic diarrhoea (Table 1). All the patients enrolled in the study were interviewed using a standard questionnaire to collect the relevant clinical information.

Eligibility criteria: All patients with a HIV sero-reactive test (result as per national guidelines) and consenting to participate in the study by providing two consecutive faecal specimens were included in the study, till the sample-size was achieved.

Two consecutive faecal specimens were collected from all the clients and subjected to examination for protozoal parasites by wet mount after concentration ${ }^{10}$. Direct smear and smears made from deposits of sedimentation were stained with Modified Acid Fast stain and examined for coccidian parasites ${ }^{10}$.

\section{Results}

The 100 HIV infected clients included in the study were between 20-50 years of age and belonged to HIV Clinical Stage 3 or 4. Of 100 patients, the protozoal parasites were detected in 25 patients. Of these 25 patients, 24 had diarrhoea, while one patient was asymptomatic.

Isospora belli was identified in $10 \%$ of HIV infected patient (Table 1). Isospora was detected in $17.24 \%$ patients with diarrhoea (in $10 \%$ of cases with acute diarrhoea, and in $21 \%$ of cases with chronic diarrhoea). Cryptosporidium was detected in $13.79 \%$ of patients with diarrhoea (in 10\% of cases with acute diarrhoea, and in $15.8 \%$ of cases with chronic diarrhoea) and in $2.38 \%$ cases without diarrhoea. Cyclospora, Microsporidia and Entamoeba histolytica were detected in $1 \%, 2 \%$ and $3 \%$ of HIV infected patients respectively.

Table 1 Protozoal Parasites detected from HIV Patients

\begin{tabular}{|l|c|c|c|c|}
\hline Parasitic Spp. & Total No. of Cases & $\begin{array}{c}\text { Cases with Acute } \\
\text { Diarrhoea }\end{array}$ & $\begin{array}{c}\text { Cases with Chronic } \\
\text { Diarrhoea }\end{array}$ & $\begin{array}{c}\text { Cases Without } \\
\text { Diarrhoea }\end{array}$ \\
\hline Isospora belli & $(\mathrm{n}=100)$ & $(\mathrm{n}=20)$ & $(\mathrm{n}=38)$ & $(\mathrm{n}=42)$ \\
\hline Cryptosporidium parvum & $10(10 \%)$ & $2(10 \%)$ & $8(21 \%)$ & 0 \\
\hline Microsporidium sp. & $9(9 \%)$ & $2(10 \%)$ & $6(15.8 \%)$ & $1(2.4 \%)$ \\
\hline Cyclospora cayetenensis & $2(2 \%)$ & 0 & $2(5.3 \%)$ & 0 \\
\hline Entamoeba histolytica & $1(1 \%)$ & 0 & $1(2.6 \%)$ & 0 \\
\hline
\end{tabular}




\section{Discussion}

The study demonstrated a very high prevalence $(25 \%)$ of protozoal parasitic infection in HIV infected individuals. The prevalence of parasitic infection was higher in patients having diarrhoea as compared to patients without diarrhoea $(41.37 \%$ vs. $2.38 \%$, ,). Isospora belli was found to be predominant cause of morbidity in symptomatic clients (Table 1). These findings are analogous to those documented in similar studies conducted in different part of the world in HIV infected individuals 11,13 , ${ }^{14}$. The prevalence of Isospora was $17.24 \%$ in patients with diarrhoea which was slightly higher as compared to the similar study from South India ${ }^{11}$. Isospora belli infections are common in patients with AIDS and chronic diarrhoea from developing countries and found to be $12-19 \%$ of patients of diarrhoea in the countries of Zambia, Haiti and Uganda. ${ }^{5}$

Cryptosporidium parvum appeared to be a second predominant parasite. Isolation of Cryptosporidium was relatively rare in our study (9.0\%) compared to other studies (30.0\%) in HIV patients ${ }^{13}$. Association of Cryptosporidium $13.79 \%$ in diarrhoeal patients in our study correlates with the study done in Chennai.11 Detection rate of Cryptosporidium oocyst found to be $2.38 \%$ in asymptomatic HIV patients. Other studies have reported Cryptosporidium as the predominant pathogen with significant association to diarrhoeal illness as well as its association with asymptomatic case. ${ }^{11}$ Occurrence of Cryptosporidium in both symptomatic and asymptomatic cases indicates high risk of infection of this parasite.

Detection rate of Cyclospora in this study found to be $1.0 \%$ in HIV patients which correlates with the study in Chennai $(0.6 \%)^{11}$ and lower compared to other similar study (11.0\%). ${ }^{13}$ Microsporidia was detected in 2 cases with chronic diarrhoea. It causes proliferate disease in immunocompromised and is usually associated with chronic diarrhoea. ${ }^{15}$

Entamoeba histolytica was detected in 3\% of cases with diarrhoea. Test to prove pathogenecity of the detected 3 species of Entamoeba histolytica could not be done, but the presence of this parasite in the AIDS patient could not be neglected otherwise. ${ }^{4}$

Difference in the incidence of intestinal protozoal parasitic infection reported by different workers ${ }^{11,13,14}$ can be attributed to the difference in geographical distribution of parasites, sanitary practices and different selection of cases. Though mixed infection is seen in AIDS patient but in our study we did not observed any such finding. Though the reasons for the same were not ascertained, this could be attributed to the limited study sample.

The present study to the best of our knowledge is the first report of the detection of opportunistic protozoal parasite in HIV patients in Saurashtra region. The rate of infection with a particular enteric parasite in HIVI AIDS patient will depend upon the endemicity of that particular parasite in the region. Laboratory support is essential to determine the carrier, latent and clinical infection. Simple stool examination with modified acid fast staining technique on a concentrated stool samples may reveal the existence of parasitic infections. The technique is economical, rapid and good for differentiating infective agents of intestinal protozoal parasites. 


\section{Conclusion}

The study enhances awareness of the prevalent opportunistic protozoal parasite in the region of Saurashtra and limits extensive evaluation or nonspecific treatment of diarrhoeal illness in HIV patients. An early and accurate diagnosis of infection will not only help in institution of specific treatment and prophylaxis (Chemoprophylaxis where ever necessary) to prevent relapse/ reoccurrence of infections in HIV patients but also in institution of various preventive measures. This will not only prolong the life of HIV infected individuals but also improve the quality of life.

\section{References}

1. Mukhopadya A, Ramakrishna B.S., Gagandeep Kang. Enteric pathogens in southern Indian HIV infected patients with and without diarrhoea.

Indian J. Med. Res (1999) 109: 80 - 89.

2. Cegieslki J.P., Ortegay R., M C Kees. Cryptosporidium, Enterocytozoon and cyclospora infection to pediatric and adult patients with diarrhoea in Tanzania. Clin. Infect Dis (1994) 24: 214- 21.

3. Janoff E N, Smith PD, and Blaser M J. Acute antibody responses to Giardia lamblia are depressed in patient with AIDS. J. Infect. DIS (1988) 157: 789 - 804.

4. Hung $C \mathrm{C}$, Chen $\mathrm{PJ}$, $H$ Sieh $S M$, Wong $\mathrm{J}$ $M$, Fang $C T$, Chang $S C$ and chen $M$. Invasive amoebiasis ; an emerging parasitic disease in patient with HIV in an area endemic for amoebic infection. AIDS (1999) 3: $2421-8$
5. Fayer R, Ungar LP. Cryptosporidium spp and cryptosporidiosis; Microbial (Rev) (1986) 50:458-83

6. Whiteside M.E. Barkunjs, Hay. Enteric coccodiosis among patients with the acquired immuno deficiency syndrome. Am.J. Trop Med Hyg (1984) 33: 1065 -72

7. Ortega $Y R$, sterling $C R$ Gilman R.H, (mava) Diaze. Cyclospora a species- a new protozoan pathogen of humans $N$. Eng $\mathrm{J}$. Med 9(1933) 320: 1308 - 1312.

8. Wurtz RM, Kokcka FE, Peterss cs. Clinical characteristics of seven cases of diarrhoea associated with a novel acid fast organism in stool J.Infec Dis (1933) 16:136 - 8

9. Shilm DR/ Cohen MT/ Eaton M An algae like organism associated with an Outbreak of prolonged diarrhoea among foreigners in Nepal. A.M.J. Trop. Med. Hyg. (1999) 45: 383- 9.

10. Monica Cheesbrough; District laboratory practice in Tropical and Wilson. Ninth Edition. (1998) Vol. 5, 208

11. Kumar SS, S ananthan, P.Laxmi.Intestinal Parasitic infection in HIV Patients with diarrhoea in Chennai., Indian $\mathrm{J}$ med. Microbial (2002) 20 (2); 88 -91

12. Pappe J.W, Verdier R1, Boney $M$ Cyclospora infection in adults infected with HIV, Clinical manifestation, Treatment \& prophylaxis. Ann Inter.Med. (1994)121:654 - 57

13. Nunez $F A$, Escoledo $A$ A prevalence of Intestinal parasites in human. AIDS patients Acto Tropica (1999) 72:125 - 30.

14. MolinaJ.M., sarcastic C. Intestinal Microsporidiosis in human immunodeficiency virus infected patient with chronic unexplained diarrhoea prevalence and biologic features. J. infects. Dis. (1993) 167: 217-21 